\title{
Repurposed Drugs as a Ray of Hope for COVID-19 Patients
}

\author{
Arshad Jawed ${ }^{1}$, Rehana Parveen ${ }^{2}$, Sajad A Dar ${ }^{1}$, Shafiul Haque ${ }^{1}$, Raju K \\ Mandal $^{1}$, Naseem Akhter ${ }^{3}$, Mohammed Y Areeshi ${ }^{1}$, Mohtashim Lohani ${ }^{4}$, \\ Ali A Rabaan ${ }^{4}$ and Mohd Wahid ${ }^{1 *}$ \\ ${ }^{1}$ Research and Scientific Studies Unit, College of Nursing and Allied Health Sciences, \\ Jazan University, Saudi Arabia \\ ${ }^{2}$ Department of Laboratory Medicine, Faculty of Applied Medical Sciences, Albaha \\ University, Saudi Arabia \\ ${ }^{3}$ Department of Emergency Medical Services, College of Applied Medical Sciences, Jazan \\ University, Saudi Arabia \\ ${ }^{4}$ Molecular Diagnostic Laboratory, Johns Hopkins Aramco Healthcare, Saudi Aramco, \\ Saudi Arabia \\ *Corresponding Author: Mohd Wahid, Research and Scientific Studies Unit, College \\ of Nursing and Allied Health Sciences, Jazan University, Saudi Arabia.
}

\author{
Received: August 11, 2020 \\ Published: April 09, 2021 \\ (c) All rights are reserved by Arshad \\ Jawed., et al
}

\begin{abstract}
The ongoing COVID-19 pandemic is as an outbreak of pneumonia caused by a novel coronavirus (nCoV). The information, knowledge and treatment about COVID-19 is in a fast gear as it is changing everyday since clinicians and researchers all over the world are trying currently available drugs like chloroquine, hydroxychloroquine, azithromycin, convalescent plasma, lopinavir- ritonavir, remdesivir etc., to eradicate this menace. Till date, the best and quick results have been shown by the use of hydroxychloroquine and azithromycin. The combination has shown almost 100\% recovery of COVID-19 patients with cautioned administration considering patient's cardiac history, if the benefits outweighs the associated risks. The convalescent plasma therapy too has shown good results for COVID-19 patients. The patients having severe respiratory problems like Acute Respiratory Distress Syndrome get resolved in 4 patients after 12 days of transfusion and 3 patients were removed from mechanical ventilation within 2 weeks of treatment. This therapy has not yet reported any adverse events though ABO blood type matching should be done before convalescent plasma transfusion from donor to receiver. Though, the number of patients used for clinical trials of hydroxychloroquine-azithromycin combination and convalescent plasma therapy were very less to prove the efficacy of these drugs for COVID-19 treatment. But the use of these drugs should be warranted when human race is facing the devastation caused by SARS-CoV-2 infection. The drug named remdesivir very recently has shown positive results as it is reducing clinical recovery time by almost 4 days or $31 \%$ when compared with placebo. The combination of other anti-viral drug lopinavir and ritonavir have not shown any positive results yet.
\end{abstract}

Keywords: COVID-19 Treatment; Hydroxychloroquine-azithromycin; Lopinavir-ritonavir; SARS-CoV-2 Infection

\section{Introduction}

COVID-19 is a pandemic haunting the human race for the last couple of months. It is a clinical disease caused by novel betacoronavirus which is also known as SARS-CoV-2. It shares simi- larity with the old SARS-CoV (79\% sequence identity) which was being held liable for a major outburst for an epidemic in the year 2002-03 [1-3]. Both SARS-CoV and SARS-CoV-2 utilizes the ACE-2 receptor for cell entry. It is suspected that higher ACE-2 expression 
may cause higher risk of SARS-CoV-2 infection [4]. The outer covering of SARS-CoV-2 is studded with a characteristic spike protein [5]. This coronavirus family also includes Middle East respiratory syndrome (MERS) CoV. Regarding the origin of this disease, it was traced back to the South China Seafood Market which is also known as 'wet' market. This market is famous for selling live or freshly slaughtered animals like snakes, poultry and bats etc. Earlier Chinese cobra was supposed to be the host but scientific community rebutted the fact saying that phylogenetic analyses showed that it originated from a bat host (88 - 96\% sequence similarity to bat coronaviruses) $[2,3,5]$.

The COVID-19 has devastating effect on the health and economic conditions of almost all the countries all over world. The number of reported cases of infection by this disease all over the world is more than 3.6 million. The reported deaths are more than 251,000 till 5-May 2020 (https://www.worldometers.info/coronavirus/). Hence, the countries all over the world are in dire need of controlling the spread of the disease by detecting the suffering patients at the earliest. The most common symptoms associated with covid-19 are fever (98\% cases), cough or stiffness in the chest $(76 \%$ cases), dyspnoea ( $55 \%$ cases) and myalgia or fatigue ( $44 \%$ cases). The patients may also show symptoms of the upper respiratory tract like coryza and sometimes haemoptysis also [6]. But most of the people infected with the virus reported to suffer from very mild symptoms and may go unnoticed. These patients showing no symptoms are known as asymptomatic carriers in the population and keep on spreading the disease without knowing the severity. Hence, there is an urgent need of the treatment for these patients to stop the further spread of the disease [7].

The scientific community has attempted many drugs used earlier for other ailments/diseases like chloroquine \& hydroxy chloroquine (old anti-malarial drugs), azithromycin (respiratory tract infection drug), convalescent plasma, lopinavir (anti-HIV drug), ritonavir (anti-HIV drug), remdesivir (used to combat ebola virus disease) etc. The current article evaluates the success and failures associated with the use of these medicines for the treatment of COVID-19.

Chloroquine, hydroxy chloroquine and azithromycin for treating COVID-19

The recent studies from China have shown that about $80 \%$ of the patients display only mild disease and overall case-fatality rate is about $2.3 \%$. The fatality rate in older patients reaches up to $8.0 \%$ (70 to 79 years age group) and $14.8 \%$ in more older patients (> 80 years of age) [8]. There is one more important factor that plays an important role is the number of asymptomatic carriers in the population, which hints that the overall mortality in the patients is over estimated. It indicates that there is a serious need to decrease the duration of virus carriage that can limit the transmission of this deadly corona virus causing COVID-19.

The scientists working at various places in the world and more specifically in severely hit COVID-19 countries have tried different treatment strategies. They have tried the repositioning of old drugs as an antiviral treatment considering their knowledge on safety, profile, side effects, posology and their interactions with other drugs $[8,9]$. Chloroquine, hydroxychloroquine and azithromycin have been tried by the scientists and found advantageous in the treatment of COVID-19 patients [10]. The mentioned paper confirmed that the COVID-19 patients were included in a single arm protocol study. The study onset in the beginning of March and ended on $16^{\text {th }}$ March-2020. The patients received $600 \mathrm{mg}$ hydroxychloroquine daily. The nasopharyngeal swab tests were done daily on these patients. Furthermore, these patients were also given azithromycin. The presence and absence of the virus in these patients on $6^{\text {th }}$ day after the start of the treatment was considered to be as end point of the study. The study showed substantial reduction of the viral carriage on $6^{\text {th }}$ day after the treatment compared to the controls. It also showed lower average carrying time than as reported in published literature. The study also showed that azithromycin added in the treatment act synergistically and was found significantly more efficient in virus elimination [10]. The results as suggested by the study after 6 days of the treatment revealed that the combination of hydroxychloroquine and azithromycin treated $100 \%$ of the patients whereas those treated with hydroxychloroquine alone showed $57.1 \%$ results. The patients kept in the control group showed only $12.5 \%$ ( $\mathrm{p}<0.001)$. Furthermore, the studies published recently have shown reduction in viral load and pulmonary recovery in the patients using hydroxy chloroquine and azithromycin [11-13].

\section{Convalescent plasma for treating COVID-19}

The COVID-19 is a pandemic with high mortality and no known cure/therapeutic agents. Therefore, the scientists are trying well known existing therapies since the spread of COVID-19. The use of convalescent plasma is one of the immunologically based forgot- 
ten therapies to treat many infectious diseases by using passive immunization technique [14]. Hence, the scientists or doctors working in China treating COVID-19 tried convalescent plasma on the patients [15]. The study was conducted in China at the department of infectious diseases, Shenzhen Third People's Hospital. The study was conducted from $20^{\text {th }}$ January-2020 to $25^{\text {th }}$ March-2020. The ABO blood types matching of the patients were done for their compatibility with the convalescent plasma donors. All the donors were asymptomatic for at least last 10 days. The antibody titer of these donors was measured for SARS-CoV-2-specific ELISA antibody and was found to be greater than 1:1000 and a neutralizing antibody titer higher than 40 . All five patients selected for convalescent plasma treatment were under mechanical ventilation and all had received antiviral agents and methylprednisolone. The age of these 5 patients was between 36 - 65 years ( 2 women). The convalescent plasma was administered between 10 and 22 days after their admission in the hospital. Different body parameters of these patients were measured after the transfusion of convalescent plasma like body temperature, SOFA (Sequential Organ Failure Assessment) score and $\mathrm{PA}_{\mathrm{O} 2} / \mathrm{FI}_{02}$. The body temperature of the patients was normalized within 3 days in 4 of 5 patients. The SOFA score was decreased in these patients whereas $\mathrm{PAO}_{2} /$ $\mathrm{FIO}_{2}$ increased within 12 days. The viral loads of these patients also got decreased and became negative within 12 days after the transfusion. These patients also showed an increase in the titer of SARS-CoV-2-specific ELISA and neutralizing antibody following the transfusion of convalescent plasma. The patients suffering from ARDS (Acute Respiratory Distress Syndrome) resolved in 4 patients after 12 days of transfusion and 3 patients were removed from mechanical ventilation within 2 weeks of treatment. Three patients were discharged from the hospital after 53, 51 and 55 days, respectively. The remaining 2 patients were stable after 37 days of convalescent plasma transfusion [15]. Hence, the current study showed the treatment to be effective and can be followed for the patients if they appear to be in critical condition. Additionally, this treatment also appears to have less side effects in comparison to other treatments followed for COVID-19 patients.

\section{Lopinavir and ritonavir for treating COVID-19}

These drugs named lopinavir and ritonavir were developed by Abbott Laboratories. They were used to inhibit an enzyme protease found in HIV; it cleaves a long protein chain which is essential for assembly of new viruses. This combination of these drugs has shown results in marmosets infected with MERS virus. It has also been tested in human patients with SARS and MERS though the results were ambiguous [16]. Recently, a new study has been published on clinical trials of lopinavir and ritonavir for the treatment of COVID-19 patients. A randomized, controlled, open-label trial was conducted in this study which involves hospitalized adult patients with confirmed SARS-CoV-2 infection. They were having an oxygen saturation $\left(\mathrm{SaO}_{2}\right)$ of $94 \%$ or less at ambient air condition. The partial pressure of oxygen $\left(\mathrm{PaO}_{2}\right)$ to the fraction of inspired oxygen $\left(\mathrm{FiO}_{2}\right)$ was less than $300 \mathrm{~mm} \mathrm{Hg}$. The total number of patients enrolled for the study were 199 in which 99 patients were assigned to the lopinavir-ritonavir group and 100 patients were kept only on standard care. One group received lopinavir-ritonavir (400 $\mathrm{mg}$ and $100 \mathrm{mg}$, respectively) twice a day for 14 days along with standard care whereas the other received only standard care.

The results as obtained during this study were not found to be encouraging. The treatment with lopinavir-ritonavir did not show any difference from standard care in parameters like hazard ratio (viz. 1.24; 95\% confidence interval $[\mathrm{CI}]$ ), the mortality rate was also found to be similar after 28 days of the treatment $19.2 \%$ vs. 25.0\%; difference). The percentages with detectable viral RNA at various time points were similar. The only parameter found positive during the complete study was that the group of patients who received lopinavir-ritonavir showed less adverse events in comparison to standard care group.

At this point of time, the combination of lopinavir-ritonavir treatment doesn't show any benefit over standard care treatment. The future clinical trials on more serious COVID-19 patients may eliminate the possibility of further treatment benefit [17].

\section{Remdesivir for treating COVID-19}

Remdesivir is a novel antiviral drug discovered by a company named Gilead Sciences. It is an adenosine nucleotide analog which incorporates itself into nascent viral RNA chains and causes their premature termination. It was developed as a treatment for Ebola virus disease and Marburg virus infections [18]. Recently, at a time of COVID-19 pandemic many drugs have been repurposed for treating the infection caused by SARS-CoV-2. There are reports for the repurposing of remdesivir as well for the treatment of COVID-19. These reports suggest that the drug is showing results in vitro against the 2019-nCoV which establishes the initial basis for clinical guidance [19]. The data shows that EC90 value of remdesivir against 2019-nCoV in Vero E6 cells was $1.76 \mu$ M. Remdesivir has also inhibited the viral infection efficiently in human 
liver cancer Huh-7 cells which is sensitive to 2019-nCoV [20]. A study was conducted at ten hospitals situated at Hubei in China. It was a randomised, double-blind, placebo-controlled, multicentre trial. This trial was conducted between $6^{\text {th }}$ February-2020 to $12^{\text {th }}$ March-2020. 158 patients were assigned to remdesivir treatment group whereas 79 patients were kept in placebo group [21]. It was observed during this trial that those patients who received remdesivir showed faster time for clinical improvement than those who were given placebo treatment. Therefore, it can be inferred from these trials that the mentioned drug is showing reduction in time for clinical improvement though larger studies involving more number of patients are required to confirm this statement. Very recently, remdesivir has been approved by US based agency named FDA saying that remdesivir reduce recovery time for COVID-19 patients by almost 4 days or $31 \%$ when compared with placebo [22] (https://www.fiercepharma.com/pharma/gilead-s-remdesivirscores-emergency-fda-nod-covid-19-days-after-big-data-reveal).

\section{Conclusion}

This article has discussed the use of various drugs like chloroquine, hydroxy chloroquine, azithromycin, convalescent plasma, lopinavir, ritonavir, remdesivir etc. The combination of hydroxy chloroquine and azithromycin proved itself to be the best drug combination for the treatment of COVID-19. This drug combination is treating COVID-19 in 6 days, which can be considered as an added advantage. But while using this combination, the physicians have to take precautions as this combination is known to affect the heart rhythm in some patients which may increase the risk of irregular heartbeat and can be life-threatening.

The use of convalescent plasma has also shown good results in treating COVID-19 patients. It has an auxiliary advantage that the transfusion of convalescent plasma does not have any side effect if the donors and receiver $\mathrm{ABO}$ blood matching has been done perfectly. Hence, it is again proving its mettle as an old agent to transfer passive immunity.

The recent studies on remdesivir have shown slight improvement in the clinical improvement time of 4 days in COVID-19 patients when compared to placebo treatment group as stated in an emergency approval letter by FDA chief. Therefore, it can also be used for the treatment of COVID-19 patients as the world is in dire need for the treatment of the disease as it is haunting the mankind economically, socially and health wise as well.
The treatment with lopinavir-ritonavir has not shown any difference from standard care in parameters like hazard ratio, mortality rate and percentages of detectable viral RNA for the patients treated with this combination of drugs. Therefore, it can be concluded that it doesn't show any benefit over standard care treatment. This notion can be strengthened further by more clinical trials using lopinavir-ritonavir for COVID-19 treatment [18].

\section{Acknowledgements}

The authors acknowledge the Deanship of Scientific Research, Jazan University, Saudi Arabia for providing the necessary support for this study.

\section{Conflicts of Interest}

The authors declare no competing interests exist.

\section{Bibliography}

1. Wu F., et al. "Complete genome characterisation of a novel coronavirus associated with severe human respiratory disease in Wuhan, China". bioRxiv (2020).

2. Zhou P., et al. "Discovery of a novel coronavirus associated with the recent pneumonia outbreak in humans and its potential bat origin". bioRxiv (2020).

3. Lu R., et al. "Genomic characterisation and epidemiology of 2019 novel coronavirus: implications for virus origins and receptor binding". Lancet (2020).

4. Guo J., et al. "Coronavirus Disease 2019 (COVID-19) and Cardiovascular Disease: A Viewpoint on the Potential Influence of Angiotensin-Converting Enzyme Inhibitors/Angiotensin Receptor Blockers on Onset and Severity of Severe Acute Respiratory Syndrome Coronavirus 2 Infection, 2020". Journal of the American Heart Association 9 (2020): e016219.

5. Zhu N., et al. "A Novel coronavirus from patients with pneumonia in China, 2019". The New England Journal of Medicine 382.8 (2020): 727-733.

6. Lake MA. "What we know so far: COVID-19 current clinical knowledge and research". Clinical Medicine 20.2 (2020): 124127.

7. Wu Z and McGoogan JM. "Characteristics of and important lessons from the coronavirus disease 2019 (COVID-19) outbreak in China: summary of a report of 72314 cases from the Chinese Center for Disease Control and Prevention". JAMA (2020). 
8. Colson P., et al. "Chloroquine for the 2019 novel coronavirus SARS-CoV-2". International Journal of Antimicrobial Agents 55.3 (2015): 105923.

9. Colson P., et al. "Chloroquine and hydroxychloroquine as available weapons to fight COVID-19". International Journal of Antimicrobial Agents 55.4 (2020): 105932.

10. Gautret P., et al. "Hydroxychloroquine and azithromycin as a treatment of COVID-19: Results of an open-label non-randomized clinical trial". International Journal of Antimicrobial Agents 56.1 (2020).

11. Gautret P., et al. "Clinical and microbiological effect of a combination of hydroxychloroquine and azithromycin in $80 \mathrm{CO}$ VID-19 patients with at least a six-day follow up: an observational study" (2020).

12. Chen Z., et al. "Efficacy of hydroxychloroquine in patients with COVID-19: results of a randomized clinical trial". medRxiv (2020).

13. Fabio ST., et al. "Hydroxychloroquine in the management of critically ill patients with COVID-19: the need for an evidence base". The Lancet Respiratory Journal 8.6 (2020): 539-541.

14. Cunningham AC., et al. "Treatment of COVID-19: old tricks for new challenges". Critical Care 24 (2020): 91.

15. Shen C., et al. "Treatment of 5 Critically Ill Patients WithCOVID-19 With Convalescent Plasma". JAMA 323.16 (2020): 15821589.

16. Kupferschmidt $\mathrm{K}$ and Cohen J. "Race to find COVID-19 treatments accelerates". Science 367.6485 (2020): 1412-1413.

17. Cao B., et al. "A Trial of Lopinavir-Ritonavir in Adults Hospitalized with Severe Covid-19. 2020". The New England Journal of Medicine (2020).

18. Warren TK., et al. "Therapeutic efficacy of the small molecule GS-5734 against Ebola virus in rhesus monkeys". Nature 531.7594 (2016): 381-385.

19. Khalili JS., et al. "Novel coronavirus treatment with ribavirin: Groundwork for an evaluation concerning COVID-19". Journal of Medical Virology (2020).

20. GX., et al. "Remdesivir and chloroquine effectively inhibit the recently emerged novel coronavirus (2019-nCoV) in vitro". Cell Research (2020): 1-3.
21. Wang Y., et al. "Remdesivir in adults with severe COVID-19: a randomised, double-blind, placebo-controlled, multicentre trial". Lancet (2020).

22. Gilead's remdesivir scores emergency FDA nod in COVID-19 days after big data reveal.

\section{Assets from publication with us}

- Prompt Acknowledgement after receiving the article

- Thorough Double blinded peer review

- Rapid Publication

- Issue of Publication Certificate

- High visibility of your Published work

Website: https://www.actascientific.com/

Submit Article: https://www.actascientific.com/submission.php Email us: editor@actascientific.com

Contact us: +919182824667 